\title{
BIBLIOGRAFÍA ESPECIALIZADA SOBRE TRADUCCIÓN E INTERPRETACIÓN: LA REVISTA MonTI (UNIVERSIDADES DE ALICANTE, JAUME I y VALÈNCIA) (2009-2015) \\ Javier Franco Aixelá \\ Universidad de Alicante
}

\section{ABSTRACT}

This bibliographic article provides an overview of MonTI (Monographs in Translation and Interpreting), a yearly academic journal born in 2009 and jointly published by the three public universities with a Translation Degree in the Valencia region of Spain.

KEY WORDS: Bibliography, Translation studies, Academic journals.

\section{RESUMEN}

Este artículo bibliográfico propone una panorámica de MonTI (Monografías de Traducción e Interpretación), una revista académica anual nacida en 2009 y publicada conjuntamente por las tres universidades públicas que ofrecen un grado en Traducción e Interpretación en la Comunidad Autónoma Valenciana.

PALABRAS CLAVE: Bibliografía, Estudios de traducción, Revistas académicas.

FECHA DE RECEPCIÓN: 21/01/2015

FECHA DE ACEPTACIÓN: 29/06/2015

PÁGINAS: 853-870 


\section{INTRODUCCIÓN}

En 2010, cuando la revista MonTI apenas acababa de nacer, tuve la oportunidad de escribir en la revista Panace@un artículo sobre nuestros objetivos e ilusiones tras dos números publicados. Con siete números ya a la vista de todo el mundo académico, lo que sigue podría considerarse en buena medida una actualización y una consolidación de lo que en aquel momento eran en esencia promesas e intenciones.

MonTI (Monografías de Traducción e Interpretación / Monografies de Traducció i d'Interpretació / Monographs in Translation and Interpreting / Monographies de Traduction et d'Interprétation / Monographien zur Translation / Monografie di Traduzione e Interpretazione) es una revista académica con carácter anual y dedicada exclusivamente a la investigación en el área de la traducción e interpretación que publicó su primer número en 2009. Los contactos que condujeron a la creación de MonTI se produjeron en 2007, aprovechando el impulso acumulado en la elaboración del programa oficial de posgrado "La traducción y la sociedad del conocimiento". Dicho programa intentaba, por una vez y sin que sirva de precedente, racionalizar la oferta universitaria española, al menos en nuestro rincón del país. De esa unión de fuerzas surgió también lo que ahora llaman sinergias y antes apenas se llamaba la confianza mutua necesaria para unir a unos cuantos irreductibles en torno a la idea de crear una revista académica anual interuniversitaria que recibiría el nombre de MonTI, acrónimo que, además de connotaciones procedentes del arte popular que lo hacen memorable, posee la virtud de ser igualmente válido para cinco y medio de los seis idiomas oficiales de la revista, algo que refuerza el multilingüismo que forma parte de la ideología básica común a nuestra disciplina.

He empezado mencionando el carácter interuniversitario de la revista porque se trata de una característica que nos resulta muy querida. Efectivamente, creemos que este tipo de iniciativas colaborativas son especialmente interesantes por tres grandes razones muy relacionadas entre sí: en primer lugar, pensamos que en el mundo de los estudios de la traducción una revista nueva y vinculada a un único departamento universitario corre el grave peligro de acabar siendo un proyecto local de muy corto alcance, probablemente no merecedor del esfuerzo que supone poner en marcha algo tan complejo como una revista académica. En este sentido, cabe argumentar que empieza a haber cierto exceso de pequeñas revistas académicas centradas en la traducción (más de 100 activas según datos de BITRA http://dti.ua.es/es/bitra/introduccion.html - a diciembre de 2014, con una veintena de ellas publicadas desde distintos rincones de España). Ante este panorama, renunciamos de partida a la idea de crear una nueva revista cajón de 
sastre y preferimos intentar dotar a la nuestra de un perfil propio que complementase de algún modo novedoso la nutrida oferta ya existente. En este sentido, creemos que elcarácter interuniversitario nos permitió reunir una masa crítica mayor de lo habitual para que la nueva revista tuviese más posibilidades de atraer contribuciones de peso y constituirse como un polo de investigación y reflexión. Las firmas de buena parte de los artículos con especialistas de primera línea parecen atestiguar que así está siendo, como podrá juzgar personalmente el lector a través de la consultadel listado final con que se cierra este artículo.

Una segunda ventaja importante de lo interuniversitario consiste en que en un contexto en el que cualquier manuscrito es por definición obra de alguien ajeno a más de dos tercios del Comité de Redacción, el proceso de selección se convierte en algo necesariamente más aséptico, razón por la que también creemos que la creación de un comité de redacción compuesto en pie de igualdad por miembros de varias universidades ayuda mucho a evitar las inevitables tentaciones endogámicas.

En tercer lugar, especialmente en tiempos de acusada crisis económica como los actuales, resulta financiera y logísticamente más viable editar una revista de calidad con versión en papel si el coste de edición, tanto en términos económicos como de esfuerzo editorial, se reparte entre varios centros universitarios. Desde el punto de vista económico, MonTI es una revista sin ánimo de lucro, un proyecto enteramente académico y muy especializado destinado casi necesariamente a generar pérdidas, al menos durante sus primeros años de andadura. Al estar financiada por sus tres universidades gestoras a partes iguales, constituye un proyecto editorial que no resulta muy oneroso, mientras que su existencia nos permite contribuir al cumplimiento de nuestra función social como universidades públicas obligadas a producir, potenciar y difundir la investigación y la generación de conocimiento.

\section{MONOGRAFÍAS DE CALIDAD}

MonTI nace como revista académica centrada en añadir nueva luz a la comprensión del fenómeno traductor (es, por tanto, una revista eminentemente teórica) y en promover el debate plural y profundo. Para alcanzar este segundo objetivo central, la revista se ha estructurado como serie de monográficos, dedicadaen cada número a un único asunto sobre el que se plasman las distintas argumentaciones de autores diversos, de modo que al final se obtiene una radiografía variada y pluralista de los distintos enfoques explicativos existentes (que, por cierto, en nuestra interdisciplina son numerosos y en muchos momentos contradictorios). Por consiguiente, MonTI no es ni la habitual revista 
académica cajón de sastre ni una revista especializada en ninguna materia concreta. Cada número aborda monográficamente un asunto de interés, lo que por una parte supone una dificultad añadida al disminuir así mucho el número de manuscritos potencialmente aceptables, pero por otra nos garantiza un proceso de debate "en vivo y en directo", con distintos enfoques sobre un mismo tema que conviven en el mismo volumen.

Hace un momento decíamos que MonTI pretende aportar su granito de luz al fenómeno traductor, objetivo para el cual la originalidad y calidad de las aportaciones resulta de todo punto fundamental. Nuestra manera de intentar garantizar dicha calidad se basa ante todo en un riguroso sistema de evaluación anónima por expertos. El proceso de selección es, de hecho, incluso un poco más complejo, ya que los editores del número reciben el artículo en primer lugar y establecen un cribaje previo de carácter esencialmente temático, destinado a garantizar la pertinencia temática y la originalidad del manuscrito en el seno de la convocatoria monográfica propuesta. En segundo lugar, cada artículo propuesto es evaluado por dos expertos en un proceso denominado de doble ciego, por el que los evaluadores desconocen la autoría del manuscrito y saben que su opinión será siempre anónima para el autor, lo que les permite expresarse con total libertad. Por supuesto, la lista de evaluadores de la revista sí es pública y aparece en la página web (http://dti.ua.es/es/monti/comites.html), mientras que la lista concreta de evaluadores de cada número, siempre sin indicar qué ha evaluado cada cual, se incluye en la versión en papel del número correspondiente.

\section{FUNCIONAMIENTO INTERNO DE LA REVISTA}

El carácter interuniversitario de MonTI también ha hecho que desde un primer momento su funcionamiento se haya caracterizado por ser eminentemente colegiado, con la democracia interna, la rotación de la dirección y la transparencia como pilares irrenunciables. Así, hemos cambiado de dirección cada dos años, con un director de cada universidad patrocinadora por bienio, al tiempo que garantizamos la continuidad del proyecto mediante la estabilidad del Comité Editorial, cuyos miembros deberían permanecer en el mismo un mínimo de cuatro años, teniendo siempre la mitad de ellos una experiencia mínima de dos años en la dirección colegiada de la revista. En pos de garantizar dicha estabilidad, tenemos también unos estatutos que describen con detalle todos los mecanismos de funcionamiento de la revista y que, por supuesto, se encuentran en los 6 idiomas oficiales y a disponibilidad de quien desee consultarlos en la web de MonTI. Uno de los aspectos destacables de dichos estatutos radica en que cualquier decisión sustancial, especialmente en lo 
referente a cambios en la revista o su dinámica editorial, deberá ser siempre tomada de manera colegiada por el Comité de Redacción. Con el fin de abrir aún más los horizontes intelectuales de la revista, a los seis miembros del Comité de Redacción procedentes de las universidades patrocinadoras de la revista, hemos sumado cuatro miembros más, externos a nuestras tres universidades, procedentes de revistas hermanas, de una universidad norteamericana y de la AIETI.

\section{SOBRE LAS VIRTUALIDADES DE LA FACETA DIGITAL DE MONTI}

La página web de MonTI, a la que ya hemos hecho un par de alusiones, constituye un componente esencial de la política de transparencia y accesibilidad internacional que intentamos que nos defina. La revista trata pues de aprovechar las enormes ventajas potenciales de la nueva sociedad digital que parece que va a caracterizar al siglo XXI y, por ello, nuestra apuesta por las virtualidades comunicativas de Internet es máxima.

Así, en primer lugar, conviene indicar que se ha hecho un esfuerzo importante para que toda la información relevante, con el máximo detalle, esté disponible en la web de la revista (http://dti.ua.es/es/monti/monti.html). De esta manera, cualquier persona con una conexión a la red podrá acceder de manera instantánea y desde cualquier rincón del mundo a una descripción completa de la revista, desde su funcionamiento interno hasta las normas para postular la edición de un número, incluyendo, por supuesto, las directrices de la revista, los comités, las convocatorias vigentes, la información para ventas o suscripciones y las normas de redacción. Además, con el objeto de que dicha información sea realmente accesible para los investigadores del mundo entero, toda ella se encuentra disponible y se publica siempre en los seis idiomas oficiales de la revista: alemán, castellano, catalán, francés, inglés e italiano. Aunque esto último nos supone un esfuerzo considerable, para nosotros resulta fundamental intentar conjugar el carácter internacional de la revista con el respeto y la potenciación de las lenguas cooficiales de la región en la que ha nacido la revista: castellano y catalán.

Por otro lado, el deseo de ponernos al alcance de todos los investigadores del área de traducción e interpretación nos ha llevado también a prolongar la apuesta cibernética hasta la doble publicación, en papel y electrónica. Así, cada número de MonTI se publica primero en papel aproximadamente cada primavera y luego, en enero del año siguiente, se pone a disposición de todos los lectores interesados de manera completa y gratuita en los repositorios de nuestras tres universidades. La edición en papel nos permite 
estar físicamente presentes en las bibliotecas universitarias y en las estanterías particulares de los expertos, algo que creemos que todavía resulta fundamental para que una revista nueva como la nuestra se dé a conocer en el mundo académico. Sin embargo, la versión electrónica tiene varias ventajas intransferibles que nos parece fundamental aprovechar. En primer lugar, nos permite a nosotros y a los autores que confían en nosotros saber que el fruto de su investigación nunca va a encontrarse descatalogado ni será difícil de conseguir. Como se ha dicho antes, representamos a universidades públicas y nos sentimos en la obligación de poner a disposición de la sociedad (que nos financia) el fruto de nuestros estudios y conocimientos. Por ello, nos comprometemos a que todo lo que se publique en MonTI esté disponible para todos mientras existan nuestras universidades y exista internet: es algo que garantizan nuestros propios centros, más allá de la supervivencia de la publicación o de la voluntad de los actuales responsables de la misma. En este sentido, resulta cuando menos curioso que un instrumento como Internet, caracterizado por la fugacidad de sus contenidos, se alce aquí como garante de accesibilidad permanente, pero así es y así lo han visto otras iniciativas similares en esto como la propia ENTRECULTURAS.

Otro aspecto que resulta muy destacable del aprovechamiento de internet consiste en que la versión digital trasciende el mero volcado de la versión en papel, puesto que también nos permite poner en marcha otra estrategia que de nuevo trata de conjugar el pluralismo lingüístico con la internacionalización. Así, por razones de espacio en la versión en papel nos hemos visto obligados a imponer límites a la pluralidad lingüística y sólo podemos admitir manuscritos elaborados en los 6 idiomas oficiales ya citados. Este límite se debe sobre todo a la enorme dificultad de encontrar expertos para la revisión externa en caso de que no existiera ningún límite de idioma. Sin embargo, una vez que un artículo ha sido admitido, no existe ninguna razón de peso para no maximizar la democracia lingüística y ofrecerle a todos los autores la posibilidad de publicar en internet una versión adicional en cualquier idioma que deseen, promocionando así todas las lenguas maternas como vehículo de conocimiento científico. Tampoco existe ningún inconveniente para, por otra parte, no intentar que todos los artículos que no se hayan publicado originalmente en inglés dispongan de una versión inglesa en la web, con lo que multiplicamos exponencial y automáticamente el alcance potencial de cualquier estudio publicado en la revista. 


\section{NÚMEROS PUBLICADOS}

Siempre con el objetivo de potenciar la pluralidad, una característica sistemática de MonTI consiste en que todos los números sean coeditados por al menos dos expertos en el asunto tratado, con al menosuno de ellos ajeno a nuestras tres universidades, intentando así traer siempre la brisa de aire intelectual fresco que puede aportar un especialista externo. A fecha de enero de 2015, MonTI ha editado ya siete números, todos los cuales se encuentran disponibles en acceso abierto en http://rua.ua.es/dspace/handle/10045/13027 y que abordan los asuntos siguientes:

MonTI 1 (2009) - Editado por María del Carmen Africa Vidal Claramonte y Javier Franco Aixelá-A (Self-)Critical Perspective of Translation Theories Una visión (auto)crítica de los estudios de traducción

MonTI 2 (2010) - Editado por Oscar Diaz Fouces \& Esther Monzó - Applied Sociology in Translation Studies - Sociologia Aplicada a la Traducció

MonTI 3 (2011) - Editado por José Santaemilia \& Luise Von Flotow - Woman and Translation: Geographies, Voices and Identities - Mujer y Traducción: Geografias, Voces e Identidades

MonTI 4 (2012)- Editado por Rosa Agost, Pilar Orero \& Elena di Giovanni Multidisciplinarity in Audiovisual Translation - Multidisciplinarietat en traducció andiovisual

MonTI 5 (2013) - Editado por Miguel Ángel Vega \& Martha Pulido - The History of Translation within Translation Studies - La Historia de la Traducción como parte de los Estudios de la Traducción

MonTI 6 (2014) - Editado por Cesáreo Calvo Rigual \& Maria Vittoria Calvi Translation and Lexicography - Traducción y Lexicografia

MonTI especial 1 (2014) - Editado por Ricardo Muñoz Martín- Minding translation - Con la traducción en mente

Adicionalmente, para el año 2015 tenemos ya dos números cerrados y en cartera, uno ordinario y otro especial, que abordan ambos la interpretación:

MonTI 7 (2015) - Editado por María Jesús Blasco Mayor y Maribel del Pozo Triviño- La interpretación en el ámbito judicial en un momento de cambio / Legal Interpreting at a Turning Point

MonTI especial 2 (2015) - Editado por Catalina Iliescu y Juan Miguel Ortega Insights in Interpreting / Reflexiones sobre la interpretación

Finalmente, conviene decir que el número para el que en este momento (primer semestre de 2015) estamos recibiendo artículos es MonTI 8 (2016), La 
traducción económica, financiera y comercial / Economic, Financial and Business Translation, editado por Daniel Gallego Hernández, Geoffrey Koby y Verónica Román Mínguez.

Como puede observarse, cada uno de los números intenta abordar un asunto de interés y actualidad en la disciplina. Deseamos y esperamos ir cubriendo poco a poco las áreas más vivas e interesantes del mundo de la traducción.

A continuación, presentamos un listado de los artículos publicados en todos los números ya impresos. Como se podrá ver, en todos los casos el artículo editorial de fondo, que constituye una panorámica crítica sobre el estado de la cuestión del asunto abordado en el número, se ha publicado en inglés y en otra de las seis lenguas oficiales de la revista. Recordamos también que la práctica totalidad de los artículos que aquí enumeramos y que no estén escritos ya en inglés se encuentran además disponibles en esta lengua franca en la versión electrónica, disponible, entre otros espacios, en: http://rua.ua.es/dspace/handle/10045/13027

Finalmente, no querría presentar este listado sin hacer alusión al carácter tremendamente plural de los autores de MonTI, con investigadores pertenecientes a decenas de universidades distintas en los apenas 6 años transcurridos desde su fundación.

- MonTI 1 (2009) - Editado por María del Carmen Africa Vidal Claramonte y Javier Franco Aixelá- A (Self-)Critical Perspective of Translation Theories - Una visión (auto)critica de los estudios de traducción.Disponible en: http:/ / rua.ua.es/dspace/handle/10045/13027

Franco Aixelá, Javier (2009). "Mon'TI, a Commitment to Rigour and Plurality" / "MonTI, una aposta pel rigor i el pluralisme" / "MonTI, un pari sur la rigueur et le pluralisme" / "Mon'TI, Wir Setzen auf Wissenschaftliche Akribie und Pluralismus" / "MonTI, una apuesta por el rigor y el pluralismo". MonTI 1, pp. 9-37. DOI: http://dx.doi.org/10.6035/MonTI.2009.1.1.

Vidal Claramonte, María del Carmen África (2009). "Rethinking Translation in the 21st Century" / "A vueltas con la traducción en el siglo XXI." MonTI 1, pp. 39-58. DOI: http://dx.doi.org/10.6035/MonTI.2009.1.2

Castro Vázquez, Olga (2009). "(Re)examinando horizontes en los estudios feministas de traducción: ¿hacia una tercera ola?" / "(Re-) examining horizons in feminist translation studies: towards a third wave" MonTI 1, pp. 59-86. DOI: http://dx.doi.org/10.6035/MonTI.2009.1.3 
Cyrus, Lea (2009). "Old Concepts, New Ideas: Approaches to Translation Shifts." MonTI 1, pp. 87-106. DOI: http://dx.doi.org/10.6035/MonTI.2009.1.4

Faber Benítez, Pamela (2009). "The Cognitive Shift in Terminology and Specialized Translation." MonTI 1, pp. 107-134. DOI: http://dx.doi.org/10.6035/MonTI.2009.1.5

Gile, Daniel (2009). "Interpreting Studies: A Critical View from Within." MonTI 1, pp. 135-155. DOI: http://dx.doi.org/10.6035/MonTI.2009.1.6

Hemmat, Amrollah (2009). "Contemporary Hermeneutics and the Role of the Self in Translation." MonTI 1, pp. 157-174. DOI: http://dx.doi.org/10.6035/MonTI.2009.1.7

Nida, Eugene Albert (2009). "Understanding the Meaning." MonTI 1, pp. 175182. DOI: http://dx.doi.org/10.6035/MonTI.2009.1.8

Shamma, Tarek (2009). "Postcolonial Studies and Translation Theory." MonTI 1, pp. 183-196. DOI: http://dx.doi.org/10.6035/MonTI.2009.1.9

- MonTI 2 (2010) - Editado por Oscar Diaz Fouces \& Esther Monzó Applied Sociology in Translation Studies - Sociologia Aplicada a la Traducció. Disponible en: http://rua.ua.es/dspace/handle/10045/13027

Wolf, Michaela (2010). "Translation 'Going Social'? Challenges to the (Ivory) Tower of Babel." MonTI 2, pp. 29-46. DOI: http://dx.doi.org/10.6035/MonTI.2010.2.2

Morris, Ruth (2010). "Court Interpreting 2009: An Undervalued and Misunderstood Profession? Or: Will Justice Speak?" MonTI 2, pp. 4779. DOI: http://dx.doi.org/10.6035/MonTI.2010.2.3

Angelelli, Claudia V. (2010). "A Glimpse into the Socialization of Bilingual Youngsters as Interpreters: The Case of Latino Bilinguals Brokering Communication for their Families and Immediate Communities." MonTI 2, pp. 81-96. DOI: http://dx.doi.org/10.6035/MonTI.2010.2.4

Valero Garcés, Carmen \& Laura Gauthier Blasi (2010). "Bourdieu y la traducción e interpretación en los servicios públicos. Hacia una teoría social" = "Bourdieu and public service interpreting and translation: towards a social theory in PSIT." MonTI 2, pp. 97-117. DOI: http://dx.doi.org/10.6035/MonTI.2010.2.5

Gouanvic, Jean-Marc (2010). "Outline of a Sociology of Translation Informed by the Ideas of Pierre Bourdieu." MonTI 2, pp. 119-129. DOI: http://dx.doi.org/10.6035/MonTI.2010.2.6

Sela-Sheffy, Rakefet (2010). "'Stars' or 'Professionals': The Imagined Vocation and Exclusive Knowledge of Translators in Israel." MonTI 2, pp. 131152. DOI: http://dx.doi.org/10.6035/MonTI.2010.2.7 
Bielsa, Esperança (2010). "The Sociology of Translation: Outline of an Emerging Field." MonTI 2, pp. 153-172. DOI: http://dx.doi.org/10.6035/MonTI.2010.2.8

Bogic, Anna (2010). "Uncovering the Hidden Actors with the Help of Latour: The 'Making' of The Second Sex." MonTI 2, pp. 173-192. DOI: http://dx.doi.org/10.6035/MonTI.2010.2.9

Fernández Iglesias, Fruela (2010). "La traducción literaria y la brecha de paralaje. Reflexiones a partir de un cuestionario piloto" / "Literary translation and the parallax gap: reflections on a pilot questionnaire." MonTI 2, pp. 193-215. http://dx.doi.org/10.6035/MonTI.2010.2.10

Cábeza i Cáceres, Cristóbal (2010). "La multitraducció als estàndards català i valencià: el cas de Harry Potter i la pedra filosofal" / "Multitranslation within the Catalan and Valencian standards: Harry Potter and the Philosopher's Stone as a case in point." MonTI 2, pp. 217-247. DOI: http://dx.doi.org/10.6035/MonTI.2010.2.11

Córdoba Serrano, María Sierra (2010). "Translation as a Measure of Literary Domination: The Case of Quebec Literature Translated in Spain (19752004)." MonTI 2, pp. 249-281. DOI: http://dx.doi.org/10.6035/MonTI.2010.2.12

Diaz Fouces, Oscar (2010). "Planificació (eco)lingüística i gestió dels intercanvis lingüístics" / "(Eco)linguistic planning and languageexchange management." MonTI 2, pp. 283-313. DOI: http://dx.doi.org/10.6035/MonTI.2010.2.13

Kuznik, Anna; Amparo Hurtado Albir \& Anna Espinal Berenguer (2010). "El uso de la encuesta de tipo social en traductología. Características metodológicas" / "The use of social surveys in translation studies: methodological characteristics." MonTI 2, pp. 315-344. DOI: http://dx.doi.org/10.6035/MonTI.2010.2.14

Tyulenev, Sergey (2010). "Is Translation an Autopoietic System?" MonTI 2, pp. 345-371. DOI: http://dx.doi.org/10.6035/MonTI.2010.2.15

- $\quad$ MonTI 3 (2011) - Editado por José Santaemilia \& Luise Von Flotow Woman and Translation: Geographies, Voices and Identities - Mujer y Traducción: Geografias, Voces e Identidades. Disponible en: http:/ /rua.ua.es/dspace/handle/10045/13027

Santaemilia Ruiz, José (2011). "Introduction: Woman and Translation: Geographies, Voices, Identities / Introducción: Mujer y traducción: Geografías, voces e identidades." MonTI 3, pp. 9-49. DOI: http://dx.doi.org/10.6035/MonTI.2011.3.1 
GodayoliNogué, Pilar (2011). "Gènere i traducció en català. Bases arqueològiques per a un estat de la qüestió" / "Gender and translation in Catalan: archaeological groundwork for assessing the state of affairs." MonTI 3, pp. 53-73. DOI: http://dx.doi.org/10.6035/MonTI.2011.3.2

Tyulenev, Sergey (2011). "Women-Translators in Russia." MonTI 3, pp. 75-105. DOI: $\underline{\text { http://dx.doi.org/10.6035/MonTI.2011.3.3 }}$

Castro Vázquez, Olga (2011). "Traductoras gallegas del siglo XX: Reescribiendo la historia de la traducción desde el género y la nación" / "Galician women translators in the 20th century: rewriting the history of translation from a gender and national approach." MonTI 3, pp. 107129. DOI: http://dx.doi.org/10.6035/MonTI.2011.3.4

Buján Otero, Patricia \& María Xesús Nogueira Pereira (2011). "La (re)escritura de los márgenes. Traducción y género en la literatura gallega" / "(Re)writing the margin: translation and gender in Galician literatura." MonTI 3, pp. 131-160. DOI: http://dx.doi.org/10.6035/MonTI.2011.3.5

Akbatur, Arzu (2011). "Turkish Women Writers in English Translation." MonTI 3, pp. 161-179. DOI: http://dx.doi.org/10.6035/MonTI.2011.3.6

Brufau Alvira, Nuria (2011). "Traducción y género: El estado de la cuestión en España" / "Translation and gender: the state of the art in Spain." MonTI 3, pp. 181-207. DOI: http://dx.doi.org/10.6035/MonTI.2011.3.7

Hassen, Rim (2011). "English Translation of the Quran by Women: The Challenges of 'Gender Balance' in and through Language." MonTI 3, pp. 211-230. DOI: http://dx.doi.org/10.6035/MonTI.2011.3.8

Braga Riera, Jorge (2011). "Women in Contemporary English Drama Translation: Enhancement and Downplay Mechanisms to Portray Golden Age Damas." MonTI 3, pp. 231-255. DOI: http://dx.doi.org/10.6035/MonTI.2011.3.9

Rivas Carmona, María del Mar (2011). "La 'visibilidad feminista' de Flora Ossette en su traducción de Woman and Labour de Olive Schreiner" / "Flora Ossette's 'feminist visibility' in her translation of Olive Schreiner's Woman and Labour." MonTI 3, pp. 257-286. DOI: http://dx.doi.org/10.6035/MonTI.2011.3.10

Stratford, Madeleine (2011). "Le sujet traduisant: un autre double du je lyrique d'Alejandra Pizarnik?" / "The translating subject: another double of Alejandra Pizarnik's lyrical I?" MonTI 3, pp. 289-333. DOI: http://dx.doi.org/10.6035/MonTI.2011.3.11 
Bibliografía especializada sobre Traducción e Interpretación. La revista MonTI...

Federici, Eleonora (2011). "Metaphors in Dialogue: Feminist Literary Critics, Translators and Writers." MonTI 3, pp. 355-376. DOI: http://dx.doi.org/10.6035/MonTI.2011.3.12

Leonardi, Vanessa \& Annarita Taronna (2011). "Translators vs Translatresses' Strategies: Ethical and Ideological Challenges." MonTI 3, pp. 377-402. DOI: http://dx.doi.org/10.6035/MonTI.2011.3.13

Sardin, Pascale (2011). 'Écrire sans honte': la sexualité féminine en question dans les traductions anglo-américaines de Passion simple, L'Événement et L'Occupation d'Annie Ernaux" / "'Writing without shame': the issue of female sexuality in the Anglo-American translations of Passion simple, L'Événement and L'Occupation by Annie Ernaux." MonTI 3, pp. 403-420. DOI: http://dx.doi.org/10.6035/Mon'TI.2011.3.14

Yu, Zhongli (2011). "Gender in Translating Lesbianism in 'The Second Sex'." MonTI 3, pp. 421-445. DOI: http://dx.doi.org/10.6035/MonTI.2011.3.15

Camus Camus, María del Carmen (2011). "Women, Translation and Censorship in the Franco Regime." MonTI 3, pp. 447-470. DOI: http://dx.doi.org/10.6035/Mon'TI.2011.3.16

- MonTI 4 (2012) - Editado por Rosa Agost, Pilar Orero \& Elena di Giovanni - Multidisciplinarity in Audiovisual Translation - Multidisciplinarietat en traducció audiovisual. Disponible

en: http://rua.ua.es/dspace/handle/10045/13027

Giovanni, Elena Di; Pilar Orero Clavero \& Rosa Agost Canós (2012). "Introduction to Multidisciplinarity in Audiovisual Translation" / "Introducció a la Multidisciplinarietat en traducció audiovisual." MonTI 4, pp. 9-37. DOI: 10.6035/MonTI.2012.4.1

Serban, Adriana (2012). "Translation as Alchemy: The Aesthetics of Multilingualism in Film." MonTI 4, pp. 39-63. DOI: http://dx.doi.org/10.6035/MonTI.2012.4.2

Maszerowska, Anna (2012). "Casting the Light on Cinema. How Luminance and Contrast Patterns Create Meanings." MonTI 4, pp. 65-85. DOI: http://dx.doi.org/10.6035/MonTI.2012.4.3

Lachat Leal, Christina (2012). "Percepción visual y traducción audiovisual: la mirada dirigida" / "Visual perception and audiovisual translation: directed visión." MonTI 4, pp. 87-102. DOI: http://dx.doi.org/10.6035/MonTI.2012.4.4

Arnáiz Urquiza, Verónica (2012). "Los parámetros que identifican el subtitulado para sordos. Análisis y clasificación." MonTI 4, pp. 103-132. DOI: http://dx.doi.org/10.6035/MonTI.2012.4.5 
McClarty, Rebecca (2012). "Towards a Multidisciplinary Approach in Creative Subtitling." MonTI 4, pp. 133-153. DOI: http://dx.doi.org/10.6035/MonTI.2012.4.6

Martínez Tejerina, Anjana (2012). "La interacción de los códigos en doblaje: juegos de palabras y restricciones visuales" / "The interaction of codes in dubbing: wordplay and visual restrictions." MonTI 4, pp. 155-180. DOI: http://dx.doi.org/10.6035/MonTI.2012.4.7

Romero Fresco, Pablo (2012). "Dubbing Dialogues... Naturally. A Pragmatic Approach to the Translation of Transition Markers in Dubbing." MonTI 4, pp. 181-205. http://dx.doi.org/10.6035/MonTI.2012.4.8

Vercauteren, Gert (2012). "A Narratological Approach to Content Selection in Audio Description. Towards a Strategy for the Description of Narratological Time." MonTI 4, pp. 207-231. DOI: http://dx.doi.org/10.6035/MonTI.2012.4.9

Igareda González, Paula (2012). "Lyrics against Images: Music and Audio Description." MonTI 4, pp. 233-254. DOI: http://dx.doi.org/10.6035/MonTI.2012.4.10

Remael, Aline (2012). "For the Use of Sound. Film Sound Analysis for AudioDescription: Some Key Issues." MonTI 4, pp. 255-276. DOI: http://dx.doi.org/10.6035/MonTI.2012.4.11

Neves, Josélia (2012). "Multi-sensory Approaches to (Audio) Describing Visual Art." MonTI 4, pp. 277-293. DOI: http://dx.doi.org/10.6035/MonTI.2012.4.12

Orero Clavero, Pilar \& Anna Vilaró Soler (2012). "Eye Tracking Analysis of Minor Details in Films for Audio Description." MonTI 4, pp. 295-319. DOI: http://dx.doi.org/10.6035/MonTI.2012.4.13

Morettini, Agnese (2012). "Profiling Deaf and Hard-of-Hearing Users of SDH in Italy: a Questionnaire-based Study." MonTI 4, pp. 321-348. DOI: http://dx.doi.org/10.6035/MonTI.2012.4.14

Jiménez Hurtado, Catalina; Claudia Seibel \& Silvia Soler Gallego (2012). "Museos para todos. La traducción e interpretación para entornos multimodales como herramienta de accesibilidad universal" / "Museums for all: translation and interpreting for multimodal spaces as a tool for universal accessibility." MonTI 4, pp. 349-383. DOI: http://dx.doi.org/10.6035/MonTI.2012.4.15

Fernández Costales, Alberto (2012). "Exploring Translation Strategies in Video Game Localisation." MonTI 4, pp. 385-408. DOI: http://dx.doi.org/10.6035/MonTI.2012.4.16 
- MonTI 5 (2013) - Editado por Miguel Ángel Vega \& Martha Pulido - The History of Translation within Translation Studies - La Historia de la Traducción como parte de los Estudios de la Traducción. Disponible en: http://rua.ua.es/dspace/handle/10045/13027

Vega Cernuda, Miguel Ángel \& Martha Lucía Pulido Correa (2013). "La historia de la traducción y de la teoría de la traducción en el contexto de los estudios de la traducción" / "The history of translation and of the theory of translation in the context of translation studies." MonTI 5, pp. 9-70. DOI: http://dx.doi.org/10.6035/MonTI.2013.1

Martino Alba, Pilar (2013). "Propuesta de didactización de contenidos de Historia de la traducción para la formación del traductor" / "A proposal for a course on the History of Translation for a humanistic translator training." MonTI 5, pp. 71-96. DOI: http://dx.doi.org/10.6035/MonTI.2013.5.2

Ordóñez López, Pilar \& José Antonio Sabio Pinilla (2013). "Contribución al estudio historiográfico de la traducción. Propuesta de un manual de lecturas guiadas y sus aplicaciones" / "A contribution towards the historiographical study of translation. A proposal for a translation historiography reader." MonTI 5, pp. 97-116. DOI: http://dx.doi.org/10.6035/MonTI.2013.5.3

Pérez Blázquez, David (2013). "Examen crítico de la bibliografía sobre la historia de la traducción en España" / "CriticalReview of theBibliographyontheHistory of Translation in Spain." MonTI 5, pp. 117-137. DOI: http://dx.doi.org/10.6035/MonTI.2013.5.4

Sánchez Galvis, Jairo (2013). "Una lectura dialectal de la historia de la traducción" / "A dialectal reading of the History of Translation." MonTI 5, pp. 139-164. DOI: http://dx.doi.org/10.6035/MonTI.2013.5.5

Wakabayashi, Judy (2013). "Evaluating Historical Views on Translation: A Case Study of Motoki Ryoei, Early Japanese Theorist." MonTI 5, pp. 165191. DOI: http://dx.doi.org/10.6035/MonTI.2013.5.6

Serrano Bertos, Elena (2013). "Un déficit documental en la historiografía de la traducción en España: consideraciones acerca del teatro (austriaco) representado y no editado" / "A deficiency of documentation in translation historiography in Spain: reflections about performed but unpublished (Austrian) theatre." MonTI 5, pp. 193-211. DOI: http://dx.doi.org/10.6035/MonTI.2013.5.7

Paloposki, Outi (2013). "Translation History: Audiences, Collaboration and Interdisciplinarity." MonTI 5, pp. 213-239. DOI: http://dx.doi.org/10.6035/MonTI.2013.5.8 
Bacardí Tomàs, Montserrat (2013). "Traduir sota la dictadura franquista, traduir clandestinament: Poesia (1944-1945) i Ariel (1946-1951)" / "Clandestine translations during Franco's dictatorship: Poesia (19441945) and Ariel (1946-1951)." MonTI 5, pp. 241-256. DOI: http://dx.doi.org/10.6035/MonTI.2013.5.9

Ruiz Casanova, José Francisco (2013). "'El arte de la traducción', según Alejandro Cioranescu" / "'El arte de la traducción,' by Alejandro Cioranescu." MonTI 5, pp. 257-270. DOI: http://dx.doi.org/10.6035/MonTI.2013.5.10

García Albero, Javier (2013). "Aspectos históricos de las traducciones y traductores del Quijote en Alemania en el siglo XX" / "Historical aspects of the translations and translators of the Quixote in Germany in the 20th century." MonTI 5, pp. 271-286. DOI: http://dx.doi.org/10.6035/MonTI.2013.5.11

Majstorovic, Gorica (2013). "Literature of the Americas in the Making: U.S. Writers and Translation in Sur, 1931-1944." MonTI 5, pp. 287-298. DOI: http://dx.doi.org/10.6035/MonTI.2013.5.12

Lafarga Maduell, Francisco (2013). "La traducción de piezas extranjeras como vía hacia la modernidad en el teatro español del siglo XVIII" / "The translation of foreign playwrights as a step towards modernity in 18th century Spanish drama." MonTI 5, pp. 299-324. DOI: http://dx.doi.org/10.6035/MonTI.2013.5.13

Lépinette, Brigitte (2013). "Traduction et terminologie. A propos de deux versions espagnoles (Madrid, 1800) de la Logique de Dumarsais" / "Translation and terminology. Apropos of two Spanish versions of Dumarsais' Logique (Madrid, 1800)" = "Traducción y terminología. A propósito de dos versiones al español de la Logique (Madrid, 1800) de Dumarsais." MonTI 5, pp. 325-347. DOI: http://dx.doi.org/10.6035/MonTI.2013.5.14

Beyer, Stefan (2013). "Los ritmos y la rima de la versificación goetheana en las versiones métricas del Fausto en español" / "Goethean rhymes and rhythms in verse translations of Faust into Spanish." MonTI 5, pp. 349363. DOI: http://dx.doi.org/10.6035/MonTI.2013.5.15

Castro Ramírez, Nayelli \& Clara Foz (2013). "La circulación de las ideas positivistas en Argentina y en México: editores y traductores (18501950)" / "Positivism on the move: translators and publishers in Mexico and Argentina from 1850 to 1950." MonTI 5, pp. 365-388. DOI: http://dx.doi.org/10.6035/MonTI.2013.5.16 
- MonTI 6 (2014) - Editado por Cesáreo Calvo Rigual \& Maria Vittoria Calvi - Translation and Lexicography - Traducción y Lexicografía. Disponible en: http://rua.ua.es/dspace/handle/10045/13027

Calvo Rigual, Cesáreo \& Maria Vittoria Calvi (2014). "Traducción y lexicografía: un diálogo necesario" / "Translation and Lexicography: A Necessary Dialogue." MonTI 6, pp. 9-62. DOI: http://dx.doi.org/10.6035/MonTI.2014.6.1

Tarp, Sven (2014). "Reflexiones sobre el papel y diseño de los diccionarios de traducción especializada" / "Reflections on the role and design of online dictionaries for specialised translation." MonTI 6, pp. 63-89. DOI: http://dx.doi.org/10.6035/MonTI.2014.6.2

Fuertes Olivera, Pedro Antonio; Sandro Nielsen \& Henning Bergenholtz (2014). "The Diccionario Inglés-Español de Contabilidad: Traducción and the Diccionario Inglés-Español de Contabilidad: Traducción de Frases y Expresiones: Two Specialised Dictionaries for Translating Terms and Collocations." MonTI 6, pp. 91-114. DOI: http://dx.doi.org/10.6035/MonTI.2014.6.3

Durán Muñoz, Isabel \& Alejandro Fernández Sola (2014). "Trandix: Herramienta proactiva para la búsqueda terminológica del traductor y su evaluación" / "Trandix: proactivetool to terminologicalsearchesbytranslators and itsassessment." MonTI 6, pp. 115-140. DOI: http://dx.doi.org/10.6035/MonTI.2014.6.4

Gallego Hernández, Daniel (2014). "Terminología y traduccióneconómicafrancés-español: evaluación de recursosterminológicos en el ámbitocontable" / "Terminology and French-Spanish business translation: evaluating terminology resources for the translation of accounting documents." MonTI 6, pp. 141-166. DOI: http://dx.doi.org/10.6035/MonTI.2014.6.5

Corpas Pastor, Gloria \& Marina Roldán Juárez (2014). "Análisis de necesidades documentales y terminológicas de médicos y traductores médicos como base para el diseño de un diccionario multilingüe de nueva generación" / "Analysis of documentary and terminological needs of doctors and medical translators as a basis for the development of a next-generation multilingual dictionary." MonTI 6, pp. 167-202. DOI: http://dx.doi.org/10.6035/Mon'TI.2014.6.6

Buendía Castro, Miriam \& Pamela Faber Benítez (2014). "Collocation Dictionaries: A Comparative Analysis." MonTI 6, pp. 203-236. DOI: http://dx.doi.org/10.6035/Mon'TI.2014.6.7 
Vaxelaire, Jean-Louis (2014). "Dictionnaires et traduction de romans contemporains." MonTI 6, pp. 237-258. DOI: http://dx.doi.org/10.6035/MonTI.2014.6.8

Sánchez Martín, Francisco Javier (2014). "Lexicografía, traducción y terminología: relaciones a partir de Della Geometria di Orontio Fineo tradotte da Cosimo Bartoli (Venetia, 1587)" / "Lexicography, translation and terminology: relationships derived from Della Geometria di Orontio Fineo tradotte da Cosimo Bartoli (Venetia, 1587)." MonTI 6, pp. 259-280. DOI: http://dx.doi.org/10.6035/MonTI.2014.6.9

- MonTI especial 1 (2014) - Editado por Ricardo Muñoz Martín - Minding translation - Con la traducción en mente. Disponible en: http://rua.ua.es/dspace/handle/10045/13027

Muñoz Martín, Ricardo (2014). "Una instantánea movida de la investigación en procesos de traducción" / "A blurred snapshot of advances in translation process research." MonTI Special Issue 1, pp. 9-84. DOI: http://dx.doi.org/10.6035/MonTI.2014.ne1.1

PACTE (2014). "First results of PACTE group's experimental research on translation competence acquisition: The acquisition of declarative knowledge of translation." MonTI Special Issue 1, pp. 85-115. DOI: http://dx.doi.org/10.6035/MonTI.2014.ne1.2

Göpferich, Susanne \& Bridgit Nelezen (2014). "The language-(in)dependence of writing skills: Translation as a tool in writing process research and writing instruction." MonTI Special Issue 1, pp. 117-149. DOI: http://dx.doi.org/10.6035/MonTI.2014.ne1.3

Alves, Fabio; José LuizGonçalves\& Karina S. Szpak (2014). "Some thoughts about the conceptual / procedural distinction in translation: A keylogging and eye-tracking study of processing effort." MonTI Special Issue 1 1, pp. 151-175. DOI: http://dx.doi.org/10.6035/MonTI.2014.ne1.4

Englund Dimitrova, Birgitta \& Elisabet Tiselius (2014). "Retrospection in interpreting and translation: Explaining the process?" MonTI Special Issue $1, \quad$ pp. 177-200. http://dx.doi.org/10.6035/MonTI.2014.ne1.5

Hvelplund, Kristian Tangsgaard (2014). "Eye tracking and the translation process: Reflections on the analysis and interpretation of eye-tracking data." MonTI Special Issue 1, pp. 201-223. DOI: http://dx.doi.org/10.6035/MonTI.2014.ne1.6 
Shreve, Gregory M.; Erik Angelone \& Isabel Lacruz (2014). "Efficacy of screen recording in the other-revision of translations: Episodic memory and event models." MonTI Special Issue 1, pp. 225-245. DOI: http://dx.doi.org/10.6035/MonTI.2014.ne1.7

Rojo López, Ana María \& Marina Ramos Caro (2014). "The impact of translators' ideology on the translation process: A reaction time experiment." MonTI Special Issue 1, pp. 247-271. DOI: http://dx.doi.org/10.6035/MonTI.2014.ne1.8

Presas Corbella, Marisa \& Celia Martín de León (2014). "The role of implicit theories in the non-expert translation process." MonTI Special Issue 1, pp. 273-302. DOI: http://dx.doi.org/10.6035/MonTI.2014.ne1.9

Apfelthaler, Matthias (2014). "Stepping into others' shoes: A cognitive perspective on target audience orientation in written translation." MonTI Special Issue 1, pp. 303-330. DOI: http://dx.doi.org/10.6035/MonTI.2014.ne1.10

Risku, Hanna (2014). "Translation process research as interaction research: From mental to socio-cognitive processes." MonTI Special Issue 1, pp. 331-353. DOI: http://dx.doi.org/10.6035/MonTI.2014.ne1.11

Ehrensberger-Dow, Maureen (2014). "Challenges of translation process research at the workplace." MonTI Special Issue 1, pp. 355-383. DOI: http://dx.doi.org/10.6035/MonTI.2014.ne1.12 